\title{
Robot-assisted rehabilitation: it is the time for utilisation in in-patient health care facilities to maintain the activity of the elderly during the COVID-19 pandemic
}

\author{
Ali Mohamed Ali Ismail[D \\ Department of Physical Therapy for Cardiovascular/Respiratory Disorder and Geriatrics, \\ Faculty of Physical Therapy, Cairo University, Giza, Egypt
}

The central goal of rehabilitation is to prevent, strengthen, restore or limit the deterioration in physical, mental, and social skills in elderly people [1]. Rehabilitation treatments usually consist essentially of multiple ongoing sessions where a therapist directs and supports the patient through a set of training activities [2]. The rehabilitation sessions are normally conducted in the different rehabilitation facilities, nursing homes, homes, and different in-patient health care facilities (IPHCF).

During the coronavirus disease 2019 (COVID-19) crisis, quarantines, limitations, and social distance are major barriers to sustaining long-term therapies for the elderly without paying attention to this issue. To avoid the risk of infection for both health care providers and patients, the requirement for long-term care services, including rehabilitation, has also been cancelled or avoided. The high rate of rapid transmission and thus death by COVID-19 could increase the risk of mental, psychological, and physical health problems, especially among comorbid chronic diseased elderly [3, 4].

Nowadays, it is not possible to provide rehabilitation for the elderly in the same manner as before the COVID-19 pandemic [5]. In nursing homes and IPHCF, many staff, workers, and residents still do not wash their hands properly, and thus spread the virus. To reduce the risk of infection for both healthcare providers and patients, the current global pandemic situation is raising the need for robot-assisted rehabilitation (RAR) devices.

To improve elderly care or to encourage independent living for the elderly and people with disabilities, digital innovations, including robots, are being increasingly used. With the aid of robotics, rehabilitative work and activities are evidence-based to have a positive effect on the emotional, social and physical aspects of the elderly [6].

For two big reasons, robots are used in rehabilitation. Robots are used as a physiotherapeutic treatment device (mainly after neurological insults including stroke [7], multiple sclerosis [8], parkinsonism [9], and spinal cord injuries [10]). Also, the use of robots in rehabilitation imposes desirable benefits in people with disabilities mainly in everyday life tasks, cognitive functions, and emotional support [7].

Robot-assisted rehabilitation devices can overcome the limitations of traditional methods of manual rehabilitation. RAR devices have the ability to reliably provide intensive rehabilitation for a longer time as in stroke compared to the traditional manual therapy. RAR devices are not impacted by the abilities and exhaustion level of the rehabilitation therapists. In the long run, without the intervention and/or presence of the rehabilitation therapist, RAR devices can perform the rehabilitation sessions allowing more frequent care and potentially reducing costs in forever-disabling conditions such as stroke and spinal cord injuries that need a forever-rehabilitation.

The maximal own effort during exercise may be stimulated from the patient during RAR sessions via the experience of entertainment gained from the in-built virtual games within the RAR systems [11]. Also, the robot's friendly aspect and its ability to catch the attention of patients help to create a fun environment during the RAR programmes [2].

The robot receives thorough details about the patient. These details are used to supply feedback to control the 
architecture of the robot to adjust itself to the requirements of every patient. On the other hand, the architecture continuously sends an updated plan to continue the rehabilitation of the patient [2].

One of the main evidence-based applied aspects of RAR devices is their ability to reduce falls in the elderly via the applied help during the different hypothetical home-settled scenarios of exercise, orientation, walking training protocols. RAR devices can attract the attention of the elderly during the rehabilitation sessions by utilizing the verbal and visual motivation properties of these devices [12].

In older residents with neurological physical disabilities after stroke and parkinsonism, the need for continuous upper-and lower-extremity exercises is vital to prevent, limit, and recover limb movements and functions. In places with a suspected application of infection control measures against COVID-19 spread such as nursing homes or IPHCF, the continuation of a supervised exercise programme may be an undesirable task by many rehabilitation therapists.

This attitude may be explained due to the risk of cross-infection that may be acquired from the continuous close contact with the elderly during the application of rehabilitation sessions. In these facilities, RAR training for the limbs provides a risk-free approach in this situation.

In exercising the older patients, RAR devices may be available in two forms, the hands-on robots (unfortunately, these robots resembles the traditional manual therapy because these types of robots make direct physical contact with the patient during aiding him in performance of exercises) or non-contact (hands-off) robots that not allow any form of direct contact to the patients [13].

To lower the risk of infection by COVID-19 during the use of hands-on robots, the use of hands-off ones - either in rehabilitation or in assistance of elderly care - is very suitable in nursing homes and IPHCF in which residents live with each other in close quarters and quarantining or socially-isolating them is very difficult.

In conclusion, with the advanced invasion of technology in all aspects of life during the COVID-19 pandemic including the health-care sector, RAR devices is a fairly-new excellent therapeutic technology that is recommended to be used in rehabilitating older residents in different IPHCF to reduce their economic burden and social problems. RAR devices can face the shortage of rehabilitation therapists in remote IPHCF during times of imposed or intentional social distance. Rehabilitation therapists or even older patients in different IPHCF hope that the endless COVID-19 crisis alarms the governments of high-income countries - or even middle- or low-income countries that seek excellence in performance in the field of health care - to find a solution to allow, manufacture, or even import the RAR devices from the countries that manufacture them, for example as Japan, not only to maintain the physical rehabilitation process or the daily activity level of elderly but also to reduce the spread of viral infection among staff workers and residents as possible.

\section{REFERENCES}

1. Stott $D$, Quinn T. Principles of rehabilitation of older people. Medicine. 2017; 45(1): 1-5, doi: 10.1016/j.mpmed.2016.10.002.

2. Martín A, Pulido JC, González JC, et al. A Framework for User Adaptation and Profiling for Social Robotics in Rehabilitation. Sensors (Basel). 2020; 20(17), doi: 10.3390/s20174792, indexed in Pubmed: 32854446.

3. Ismail AMA. Cancelled elderly exercise sessions during the COVID-19 crisis: can physical therapists help from their homes? Eur J Physiother. 2020; 22(4): 235, doi: 10.1080/21679169.2020.1775293.

4. Ismail AA. Online exercise rehabilitation to stable COPD patients during the second COVID wave: are physiotherapists able to help? Advances Rehabilitation. 2020; 34(4): 48-49, doi: 10.5114/ areh.2020.101592.

5. De Biase S, Cook L, Skelton DA, et al. The COVID-19 rehabilitation pandemic. Age Ageing. 2020; 49(5): 696-700, doi: 10.1093/ ageing/afaa118, indexed in Pubmed: 32470131.

6. Melkas H, Hennala L, Pekkarinen S, et al. Impacts of robot implementation on care personnel and clients in elderly-care institutions. Int J Med Inform. 2020; 134: 104041, doi: 10.1016/j. ijmedinf.2019.104041, indexed in Pubmed: 31838291.

7. Fazekas G. Robotics in rehabilitation: successes and expectations. Int J Rehabil Res. 2013; 36(2): 95-96, doi: 10.1097/ MRR.0b013e32836195d1, indexed in Pubmed: 23603805.

8. Straudi S, Fanciullacci C, Martinuzzi C, et al. The effects of robot-assisted gait training in progressive multiple sclerosis: a randomized controlled trial. Mult Scler. 2016; 22(3): 373-384, doi: 10.1177/1352458515620933, indexed in Pubmed: 26658817.

9. Smania N, Picelli A, Geroin C, et al. Robot-assisted gait training in patients with Parkinson's disease. Neurodegener Dis Manag. 2013; 3(4): 321-330, doi: 10.2217/nmt.13.34.

10. Nam KiY, Kim HJ, Kwon BS, et al. Robot-assisted gait training (Lokomat) improves walking function and activity in people with spinal cord injury: a systematic review. J Neuroeng Rehabil. 2017; 14(1): 24, doi: 10.1186/s12984-017-0232-3, indexed in Pubmed: 28330471.

11. Xie S. Advanced robotics for medical rehabilitation. Springer Tracts Advanced Robotics. 2016, doi: 10.1007/978-3-319-19896-5.

12. Aguirre A, Casas J, Céspedes N, et al. Feasibility study: Towards Estimation of Fatigue Level in Robot-Assisted Exercise for Cardiac Rehabilitation. In 2019 IEEE 16th International Conference on Rehabilitation Robotics (ICORR). 2019: 911-916, doi: 10.1016/j. mpmed.2016.10.002.

13. Gockley R, Matarić M. Encouraging physical therapy compliance with a hands-Off mobile robot. Proceeding of the 1st ACM SIGCHI/ /SIGART conference on Human-robot interaction - HRI '06. 2006: 150-155, doi: 10.1145/1121241.1121268. 